\title{
Biannual Treatment of Preschool Children with Single Dose Azithromycin to Reduce Mortality: Impact on Azithromycin Resistance in the MORDOR Trial in Tanzania
}

\author{
Evan M. Bloch, ${ }^{1 \star}$ Christian L. Coles, ${ }^{2}$ Mabula Kasubi, ${ }^{3}$ Jerusha Weaver, ${ }^{4}$ Zakayo Mrango, ${ }^{5}$ Beatriz Munoz, ${ }^{4}$ Thomas M. Lietman, ${ }^{6}$ \\ and Sheila K. West ${ }^{4}$ \\ ${ }^{1}$ Department of Pathology, Johns Hopkins School of Medicine, Baltimore, Maryland; ${ }^{2}$ Infectious Disease Clinical Research Program, Uniformed \\ University of the Health Sciences, Bethesda, Maryland; ${ }^{3}$ Department of Microbiology, Muhimbili University of Health and Allied Sciences, Dar es \\ Salaam, Tanzania; ${ }^{4}$ Dana Center for Preventive Ophthalmology, Johns Hopkins School of Medicine, Baltimore, Maryland; ${ }^{5}$ National Institute for \\ Medical Research, Kilosa, Tanzania; ${ }^{6}$ Francis I Proctor Foundation, University of California, San Francisco, San Francisco, California
}

\begin{abstract}
The Macrolides Oraux pour Réduire les Décès avec un Oeil sur la Résistance study showed that administration of biannual, single-dose azithromycin to preschool children reduces mortality. We sought to evaluate its impact on azithromycin resistance. Thirty randomly selected communities in Kilosa district, Tanzania, were randomized to receive 6monthly single-dose azithromycin $(\sim 20 \mathrm{mg} / \mathrm{kg})$ versus placebo treatment of children aged 1-59 months. From each community, 40 children (aged 1-59 months) were randomly selected at baseline, 12 and 24 months. Isolation and resistance testing of Streptococcus pneumoniae and Escherichia coli were evaluated using nasopharyngeal and rectal swabs, respectively. The carriage prevalence and the proportion of azithromycin-resistant isolates were determined using disk diffusion. At baseline, the characteristics of the randomly selected children were similar by treatment arms. Both at baseline and in annual cross-sectional surveys, rates of $S$. pneumoniae and $E$. coli isolation between treatment arms were similar. The proportions of azithromycin-resistant $S$. pneumoniae isolates in the children in communities treated with azithromycin versus placebo at baseline, 12 months, and 24 months were $26.5 \%(18.1 \% ; P=0.26), 26.8 \%(16.5 \% ; P=$ $0.29)$, and $13.4 \%(17.0 \% ; P=0.57)$, respectively. The proportions of azithromycin-resistant $E$. coli isolates at baseline, 12 months, and 24 months in the azithromycin (versus placebo) arms were $14.9 \%(18.9 \% ; P=0.16), 21.5 \%(16.6 \% ; P=$ $0.10)$, and $14.9 \%(14.7 \% ; P=0.95)$, respectively. Over the 24 months, the mean treatment coverage for the azithromycin and placebo was $76.9 \%$ and $74.8 \%$, respectively $(P=0.49)$. Biannual administration of single-dose azithromycin to children did not appear to result in excess azithromycin resistance in S. pneumoniae and $E$. coli isolates over 24 months of follow-up.
\end{abstract}

\section{INTRODUCTION}

Azithromycin is a versatile antibiotic that is used to treat a variety of infectious diseases that are common in lowresource settings. ${ }^{1-4}$ Mass drug administration (MDA) of azithromycin has also been integral to the ongoing elimination strategy for trachoma. ${ }^{5}$ In the recent Macrolides Oraux pour Réduire les Décès avec un Oeil sur la Résistance (MORDOR, clinicaltrials.gov \#NCT02048007) trial, biannual administration of single-dose azithromycin to preschool children was shown to reduce all-cause mortality compared with placebo. ${ }^{6}$ If this were to be adopted as a public health intervention, the emergence of bacterial resistance to azithromycin and other macrolides would become a concern. This is particularly the case with Streptococcus pneumoniae. In nationwide hospital surveys in the United States, a quarter to almost a third of S. pneumoniae isolates have been shown to be resistant to azithromycin. $^{7,8}$ In the Tanzania and Malawi arms of the MORDOR trial, significant protective effects against mortality were not observed. The potential contribution of increased resistance to this lack of effect is unknown.

Studies that have evaluated bacterial resistance to azithromycin following biannual MDA for trachoma control have reported variable findings. ${ }^{9-14}$ In treatment-naïve populations in which entire communities underwent MDA, resistance developed following a single round of azithromycin but was short-lived. ${ }^{11,15}$ However, when MDA was conducted repeatedly, resistance was slow to revert to pretreatment levels

*Address correspondence to Evan M. Bloch, Department of Pathology, Johns Hopkins University, 600 N. Wolfe St./Carnegie 446 D1, Baltimore, MD 21286. E-mail: ebloch2@jhmi.edu following cessation. ${ }^{16}$ In the Tanzanian site of the MORDOR trial, we hypothesized that the proportion of azithromycinresistant isolates would be greater at 12 and 24 months in children who reside in communities that are randomized to azithromycin as compared with those who reside in communities that are randomized to receive placebo. Therefore, we sought to compare the proportion of azithromycin-resistant isolates of S. pneumoniae and Escherichia coli from children in the communities randomized to azithromycin versus placebo, comparing the proportions at 12 and 24 months of follow-up. ${ }^{17-19}$

\section{MATERIALS AND METHODS}

Overview. A cluster-randomized, placebo-controlled, doublemasked clinical trial was conducted in 30 communities in Kilosa district, Tanzania (January 2015-August 2017), as part of the MORDOR trial. This MORDOR morbidity study, which was performed in parallel to the MORDOR mortality trial, sought to evaluate the effect-in children-of biannual, single-dose azithromycin (compared with placebo) on the proportion of azithromycin-resistant isolates of $S$. pneumoniae and $E$. coli that were recovered from nasopharyngeal and rectal swabs, respectively.

Eligibility. All communities located in Kilosa district that had at least 20 children aged 1-59 months during a baseline census were eligible to participate in the trial (Figure 1). The communities were drawn from the same pool as the MORDOR mortality study. ${ }^{6}$ At each survey time point, all 15 communities in each arm participated. Within each community, 40 children, aged 1 and 59 months, who lived in the randomly selected community at the time of the survey and had a guardian who was capable of providing consent, were randomly selected to participate. Participation rates are shown in Figure 1. If a 


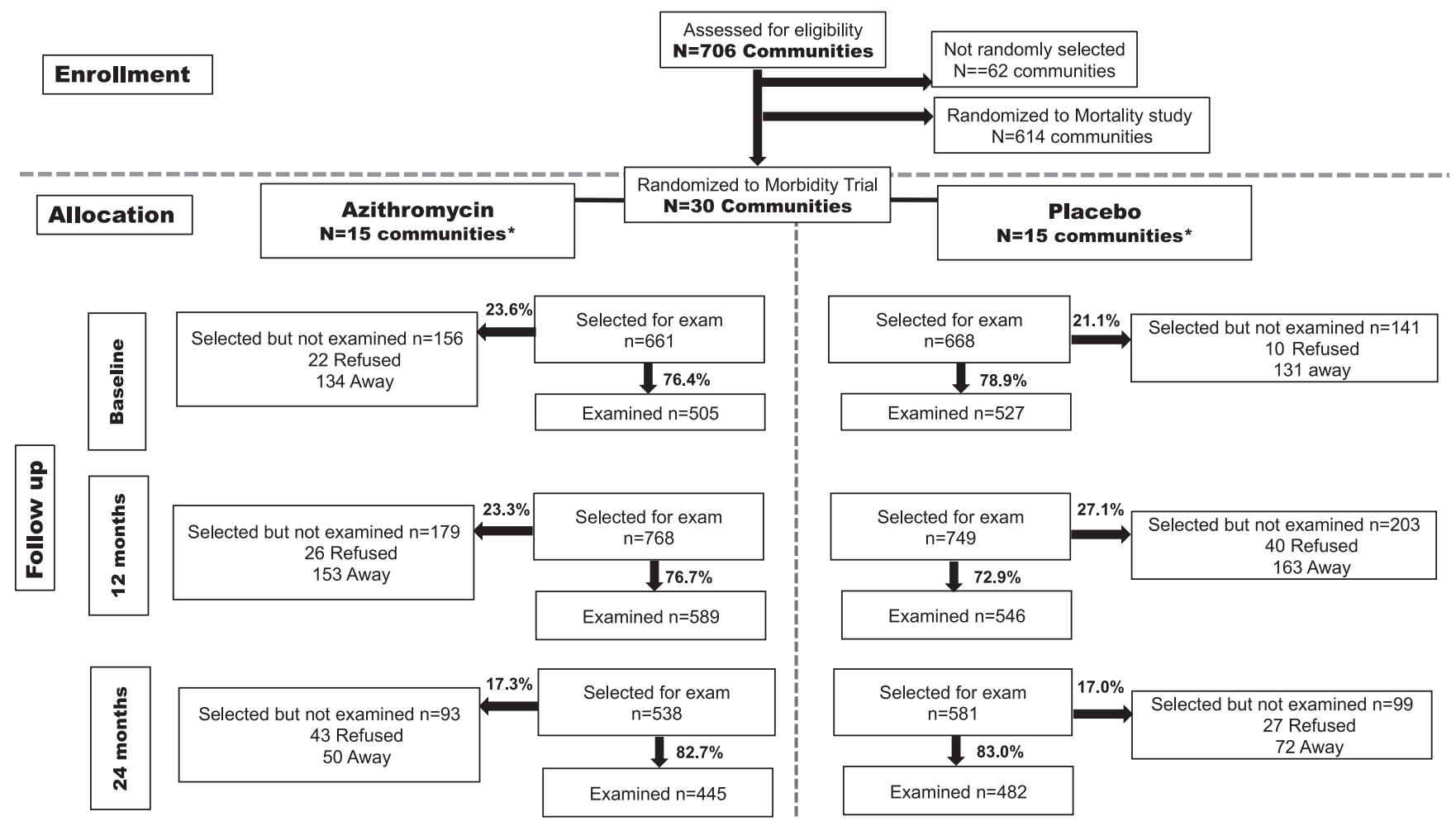

FIGURE 1. CONSORT diagram. *None of the participating communities were lost to follow-up.

community had less than 40 eligible children, all the children were selected to participate.

Randomization and masking. The lead statistician generated the randomization sequence for the morbidity study, using a series of 6 letters with a 1:1 allocation; this was implemented by the Tanzanian study team. The trial was double-masked such that the treatment assignment was unknown to the participants and study teams. Only the lead statistician who performed the random assignment was unmasked.

Intervention. The intervention was azithromycin ( 20 mg/ $\mathrm{kg}$ ) or placebo (Pfizer, New York, NY); both azithromycin and placebo were supplied in identical containers and were indistinguishable in appearance and taste. The treatments were administered every 6 months for 24 months.

Primary outcome. Proportion of azithromycin-resistant isolates of $S$. pneumoniae and $E$. coli obtained from nasopharyngeal and rectal swabs, respectively, in randomly selected children aged 1-59 months at 12 and 24 months.

Specimen collection. In each community, specimens were collected at baseline, 12 months (after two rounds of intervention), and 24 months (after four rounds of intervention). A trained field team collected nasopharyngeal and rectal swabs for culture, before the upcoming intervention. Each nasopharyngeal specimen for S. pneumoniae was obtained using a sterile, rayon-tipped swab (Puritan calcium alginate swabs, Fisher Scientific, Hampton, $\mathrm{NH}$ ). The swab was inserted in media containing skim milk, tryptone, glucose, and glycerin (STGG) and stored at $-20^{\circ} \mathrm{C}$ pending shipment on dry ice to the Muhimbili National Hospital's microbiology laboratory (MHML), within 1 month of collection. Rectal specimens for E. coli were collected using sterile cotton swabs, placed into Amies transport media and stored at $-20^{\circ} \mathrm{C}$ for up to 1 -month pending shipment on dry ice to the MHML for isolation and resistance testing. Pilot testing with swabs inoculated with E. coli showed that the organisms were still viable after storage at $-20^{\circ} \mathrm{C}$ for approximately 7 weeks.

Laboratory procedures. For S. pneumoniae isolation, swabs were inoculated onto blood agar containing 5\% sheep blood and $5 \mathrm{mg} / \mathrm{L}$ gentamicin (Becton Dickinson, Cockeysville, MD) and then incubated at $37^{\circ} \mathrm{C}$ in $5 \% \mathrm{CO} 2$ for $18-24$ hours. Colonies were confirmed by optochin disk (Taxo, Becton Dickinson) inhibition or bile solubility testing. A pneumococcal reference strain was used for quality control. Culture-positive specimens were subcultured and frozen in STGG at $-70^{\circ} \mathrm{C}$ pending antibiotic susceptibility testing.

The Kirby-Bauer disk diffusion method was used to test S. pneumoniae isolates for sensitivity to azithromycin $(15 \mu \mathrm{g}$ disk) on Mueller-Hinton media with 5\% lysed sheep blood. Inhibitory zone diameters (ZDs) were used to classify isolates as "sensitive" (ZD > $18 \mathrm{~mm}$ ), "intermediately resistant" $\mathrm{ZD} \geq$ $14 \mathrm{~mm}$ but $\leq 17 \mathrm{~mm}$ ), or "resistant" (ZD $\leq 13 \mathrm{~mm}$ ), according to the 2018 Clinical and Laboratory Standards Institute (CLSI) recommendations. ${ }^{20}$

For E. coli, swabs were streaked on MacConkey agar and cultured overnight at $37^{\circ} \mathrm{C}$. Up to three lactose-fermenting colonies were inoculated into nutrient agar stabs and grown overnight at $37^{\circ} \mathrm{C}$ followed by storage at room temperature. Indole-positive, citrate-negative isolates were considered to be $E$. coli. Break points to define macrolide resistance in $E$. coli have not been established. Therefore, azithromycin resistance was defined as a ZD $<10 \mathrm{~mm}$; this resistance cutoff was based on a prior study of $E$. coli resistance ${ }^{21}$ and was estimated using a receiver-operator characteristic curve.

Data management and statistical analyses. Data were analyzed at each cross-sectional time point, examining the 
proportion of children with isolates and the proportion of isolates that had resistant organisms, as defined earlier. Descriptive statistics were stratified by the treatment arm. Crosssectional differences in the proportion of isolates that were resistant were tested initially using logistic models with the arm as the independent predictor. The generalized estimating equations approach, with an independent correlation structure, was used to account for clustering of isolates/resistant isolates within the same community. Data were analyzed with SAS version 9.4 software (SAS, Raleigh, NC). Intermediate and resistant categories were combined and also presented. Coverage with azithromycin/placebo treatment of all children aged 1-59 months in the communities was calculated as follows: the proportion of children treated was calculated for each community, and coverage by the treatment arm was estimated as the mean of the community proportion for communities in that arm. Mean coverages and 95\% Cls are reported.

Sample size calculation. Given a baseline resistance of $12 \%,{ }^{12}$ and an intra-class correlation coefficient of approximately $0.05,{ }^{22}$ we anticipated approximately $80 \%$ power to detect a difference in the proportion of isolates with a resistance of $18 \%$ (i.e., comparing 12-30\%), assuming at least eight children per community with isolates.

Ethical review and trial oversight. Ethical approval was obtained from the Tanzanian National Institute for Medical Research and the institutional review boards of the Johns Hopkins School of Medicine and the University of California San Francisco. Children were included in the study on the basis of documented written informed consent from guardians. The study is registered at clinical trials.gov (NCT02048007). A data and safety monitoring committee provided trial oversight.

Role of the funding source. The study sponsor had no role in the study design, the collection, analysis, and interpretation of the data, in the manuscript preparation or the decision to submit the paper for publication.

\section{RESULTS}

At baseline, the demographic and clinical characteristics of the children and rates of isolation of $S$. pneumoniae and $E$. coli between the treatment arms were similar and not significantly different (Table 1).

TABLE 1

Baseline characteristics in randomly selected children by the community treatment arm

\begin{tabular}{|c|c|c|c|}
\hline \multirow[b]{2}{*}{ Characteristic } & \multicolumn{2}{|c|}{ Arm } & \multirow[b]{2}{*}{$\begin{array}{c}P- \\
\text { value }\end{array}$} \\
\hline & $\begin{array}{l}\text { Azithromycin, } \\
\qquad \begin{array}{l}N 05\end{array}\end{array}$ & $\begin{array}{l}\text { Placebo, } \\
N=527\end{array}$ & \\
\hline Age (months), mean (SD) & $27.3(16.1)$ & $28.1(15.9)$ & 0.36 \\
\hline Female, $n(\%)$ & $246(48.7)$ & 263 (49.9) & 0.69 \\
\hline $\begin{array}{l}\text { Used drugs to treat infection } \\
\text { previous } 14 \text { days, } n(\%)\end{array}$ & $117(23.2)$ & $173(32.9)$ & 0.06 \\
\hline Child is coughing, $n(\%)$ & $177(35.0)$ & $172(32.8)$ & 0.63 \\
\hline $\begin{array}{l}\text { Child has difficulty breathing, } \\
n(\%)\end{array}$ & $8(1.6)$ & $4(0.8)$ & 0.21 \\
\hline $\begin{array}{l}\text { Respiratory rate } \\
\text { (breaths/minute), mean (SD) }\end{array}$ & $37.7(4.1)$ & $37.6(3.5)$ & 0.66 \\
\hline Child has diarrhea, $n(\%)$ & $46(9.1)$ & $57(10.8)$ & 0.46 \\
\hline Child has fever, $n(\%)$ & $35(6.9)$ & $35(6.7)$ & 0.88 \\
\hline \multicolumn{4}{|l|}{ Laboratory results } \\
\hline $\begin{array}{l}\text { Streptococcus pneumoniae } \\
\quad \text { isolated, } n(\%)\end{array}$ & 219 (43.5) & $226(43.0)$ & 0.90 \\
\hline Escherichia coli isolated, $n$ (\%) & $310(61.6)$ & $324(61.5)$ & 0.99 \\
\hline
\end{tabular}

The average treatment coverage in the communities that received azithromycin was $76.9 \%$. Average coverage of the communities was significantly different between the two arms only at the 12-month treatment round, where there was higher coverage in communities in the azithromycin arm $(77.2 \%$ versus $64.8 \%$ ) (Table 2).

At 24 months, over half the children in both arms took the drug as randomized for at least three of four of treatment cycles (Table 3). In the annual cross-sectional surveys, rates of $S$. pneumoniae and $E$. coli carriage between treatment and placebo arms were similar. However, rates of isolation declined overtime (Table 3). The respective rates of E. coli isolation in the children in the azithromycin arm were $61.6 \%$, $39.6 \%$, and $58.9 \%$ compared with $61.5 \%$, $43 \%$, and $43.2 \%$ in the placebo arm.

An excess in the proportion of azithromycin-resistant $S$. pneumoniae and $E$. coli isolates was not found at baseline, 12 months, or 24 months (Table 4): the respective proportions of azithromycin-resistant $S$. pneumoniae isolates in azithromycin versus placebo arms at baseline, 12, and 24 months were $26.5 \%$ versus $18.1 \%(P=0.26), 26.8 \%$ versus $16.5 \%(P=0.29)$, and $13.4 \%$ versus $17.0 \%(P=0.57)$. The proportions of azithromycin-resistant isolates of $E$. coli at baseline, 12, and 24 months in the azithromycin versus placebo arms were $14.9 \%$ versus $18.9 \%(P=0.16), 21.5 \%$ versus $16.6 \%(P=0.10)$, and $14.9 \%$ versus $14.7 \%(P=0.95)$, respectively (Table 4).

\section{DISCUSSION}

The aggregate findings from the MORDOR trial showed that targeted biannual administration of single-dose azithromycin reduces mortality in children younger than 5 years. ${ }^{6}$ However, a reduction in mortality was not seen in the Tanzanian (also Malawian) arm of the MORDOR trial. This raised the question as to whether emergence of antibiotic resistance could have offset any potential morbidity or mortality benefit in these sites. Concern over azithromycin resistance is not limited to bacteria for which it is known to be active (e.g., S. pneumoniae), rather antibiotic selection pressure can extend to nontarget bacteria such as $E$. coli. ${ }^{21}$ This is well established, particularly in poor-resource settings. ${ }^{23,24}$ Commensal bacteria can also serve as important reservoirs for resistant bacteria. ${ }^{25,26}$ We hypothesized that children in the villages randomized to receive azithromycin would have significantly more isolates resistant to azithromycin than children in the villages randomized to receive the placebo. However, neither an excess in the proportion of azithromycin-resistant $S$. pneumoniae and $E$. coli isolates nor an increase in resistance overtime were found at either 12 or 24 months.

TABLE 2

Treatment coverage in 30 communities randomized to azithromycin vs. placebo

\begin{tabular}{lccr}
\hline & \multicolumn{2}{c}{ Average community coverage* } & \\
\cline { 2 - 3 } \multicolumn{1}{c}{ Period } & $\begin{array}{c}\text { Azithromycin, } \\
\text { mean }(95 \% \mathrm{Cl})\end{array}$ & $\begin{array}{c}\text { Placebo, } \\
\text { mean }(95 \% \mathrm{Cl})\end{array}$ & $P$-value \\
\hline Baseline & $78.1(70.0-86.6)$ & $79.6(72.5-86.6)$ & 0.78 \\
6 months & $75.0(67.0-83.0)$ & $71.7(64.4-79.1)$ & 0.21 \\
12 months & $77.2(71.2-82.6)$ & $64.8(51.2-75.8)$ & 0.04 \\
18 months & $77.4(72.1-82.8)$ & $76.2(69.4-83.0)$ & 0.76 \\
\hline * Estimated as the mean of the proportion treated in each community.
\end{tabular}


TABLE 3

Characteristics of randomly selected children at 12 and 24 months (post two and four rounds of treatment)

\begin{tabular}{|c|c|c|c|c|c|c|}
\hline \multirow[b]{2}{*}{ Characteristic } & \multicolumn{2}{|c|}{ 12-month arm } & \multirow[b]{2}{*}{$P$-value } & \multicolumn{2}{|c|}{ 24-month arm } & \multirow[b]{2}{*}{$P$-value } \\
\hline & Azithromycin, $N=589$ & Placebo, $N=546$ & & Azithromycin, $N=445$ & Placebo, $N=482$ & \\
\hline Age (months), mean (SD) & $25.5(14.1)$ & $26.1(15.5)$ & 0.51 & $29.7(16.1)$ & $26.6(16.6)$ & 0.03 \\
\hline Female, $n(\%)$ & $297(50.4)$ & $261(47.8)$ & 0.30 & $226(50.8)$ & $228(47.3)$ & 0.34 \\
\hline \multicolumn{7}{|l|}{ Laboratory results } \\
\hline $\begin{array}{l}\text { Streptococcus pneumoniae } \\
\quad \text { isolated, } n(\%)\end{array}$ & 97 (16.5) & $97(17.8)$ & 0.78 & $67(15.1)$ & $53(11.0)$ & 0.26 \\
\hline Escherichia coli isolated, $n$ (\%) & $233(39.6)$ & $235(43.0)$ & 0.54 & $262(58.9)$ & $272(43.2)$ & 0.71 \\
\hline \multicolumn{7}{|c|}{ Number of previous visits during which the child received the study drug } \\
\hline None, $n(\%)$ & $102(17.3)$ & $95(17.4)$ & 0.97 & $32(7.2)$ & $44(9.1)$ & 0.33 \\
\hline One, $n(\%)$ & $160(27.2)$ & $146(26.7)$ & & $49(11.0)$ & 54 (11.2) & \\
\hline Two, $n(\%)$ & 327 (55.5) & 305 (55.9) & & 79 (17.8) & $88(18.2)$ & \\
\hline Three, $n(\%)$ & - & - & - & $103(23.1)$ & $134(27.7)$ & \\
\hline Four, $n(\%)$ & - & - & - & $182(40.9)$ & 163 (33.8) & \\
\hline
\end{tabular}

Most studies that have reported on azithromycin resistance in S. pneumoniae isolates have been conducted in the context of trachoma in which all residents in the communities have undergone azithromycin treatment, although resistance testing may be confined to children. Most studies show an increase in azithromycin-resistant strains of $S$. pneumoniae immediately following MDA. In treatment naïve populations, a single round of mass treatment is followed by an increase in resistance that subsides after a few months. ${ }^{9}$ One study in Tanzania observed nearly absent resistance at 6 months following a single round of $\mathrm{MDA}^{9}$ However, when multiple rounds of MDA have been given, the decline is not as rapid. ${ }^{10,13}$ One study evaluated azithromycin resistance in fecal $E$. coli in eight communities following a single round of MDA in a district that had previously undergone multiple rounds of MDA; although carriage prevalence of resistant strains at baseline was similar between children in newly treated and untreated communities, the carriage of resistant isolates increased from $21 \%$ to $61 \%$ at 1 month and was still elevated $(31 \%)$ at 6 months in the newly treated communities. ${ }^{21}$

Studies of antibiotic resistance where administration of azithromycin has been targeted to children are few. In one small randomized trial in Ethiopia, children aged 1-10 years underwent 3-monthly single-dose azithromycin versus no treatment for 1 year. ${ }^{12}$ The baseline mean proportion of azithromycin-resistant isolates of $S$. pneumoniae in the treatment arm was $6.3 \%$, which increased to $62.3 \%$ at 12 months. Although baseline resistance was not reported in the untreated communities, $11.6 \%$ of $S$. pneumoniae isolates were resistant at 12 months. ${ }^{12}$

Several possible explanations were considered as to why we did not find an increase in resistant isolates. The most likely is that unlike the trachoma trials that treat everyone in the community, we only treated children aged 1-59 months, who represent a small ( $15 \%$ or less) fraction of the community. This leaves a larger population pool with susceptible strains that are available to repopulate the nasopharynx of the treated children. Not only was there no increase in resistance by treatment assignment but also the proportion of azithromycin-resistant S. pneumoniae isolates did not increase from baseline, and, if anything, the point estimate decreased by 24 months, following four rounds of drug administration. Had there been a lasting increase in resistance (i.e., as a result of multiple rounds of azithromycin treatment of children in those communities), the 24-month survey would have been the most informative.

We acknowledge that with follow-up visits at 1 year and 2 years, we were not able to detect evidence of a transient increase in resistance that might have occurred immediately

TABLE 4

Percentage of Streptococcus pneumoniae and Escherichia coli isolates resistant to azithromycin by the study arm and time of survey

\begin{tabular}{|c|c|c|c|c|}
\hline \multirow[b]{3}{*}{ Survey time } & \multirow[b]{3}{*}{ Characteristic } & \multicolumn{2}{|c|}{ Arm } & \multirow[b]{3}{*}{$P$-value ${ }^{*}$} \\
\hline & & \multirow{2}{*}{$\frac{\text { Azithromycin }}{\begin{array}{c}\text { Proportion of resistant } \\
\text { isolates }(95 \% \mathrm{Cl})^{\star}\end{array}}$} & \multirow{2}{*}{$\frac{\text { Placebo }}{\begin{array}{c}\text { Proportion of resistant } \\
\text { isolates }(95 \% \mathrm{Cl})^{\star}\end{array}}$} & \\
\hline & & & & \\
\hline \multirow[t]{5}{*}{ Baseline } & S. pneumonia $(N=219,226) \dagger$ & - & - & - \\
\hline & Intermediate & 9.1 & 6.6 & \\
\hline & Resistant & 17.4 & 11.5 & \\
\hline & Intermediate/resistant & $26.5(16.5-39.6)$ & $18.1(10.9-28.6)$ & 0.26 \\
\hline & E. coli resistance $(N=309,323) \dagger$ & $14.9(11.6-18.9)$ & $18.9(14.9-23.6)$ & 0.16 \\
\hline \multirow[t]{5}{*}{12 months } & S. pneumonia $(N=97,97) \dagger$ & - & - & - \\
\hline & Intermediate & 8.2 & 2.1 & \\
\hline & Resistant & 18.6 & 14.4 & \\
\hline & Intermediate/resistant & $26.8(13.9-45.4)$ & $16.5(8.1-30.7)$ & 0.29 \\
\hline & E. coli resistance $(N=233,235) \dagger$ & $21.5(17.0-26.7)$ & $16.6(13.5-20.3)$ & 0.10 \\
\hline \multirow[t]{5}{*}{24 months } & S. pneumonia $(N=67,53) \dagger$ & & & \\
\hline & Intermediate & 0.0 & 3.8 & \\
\hline & Resistant & 13.4 & 13.2 & \\
\hline & Intermediate/resistant & $13.4(8.4-20.8)$ & $17.0(8.2-31.8)$ & 0.57 \\
\hline & E. coli resistance $(N=262,272) \dagger$ & $14.9(10.8-20.0)$ & $14.7(10.9-20.1)$ & 0.95 \\
\hline
\end{tabular}

* Using the generalized estimated equation approach with an independent correlation structure to account for clustering within the same community.

† Number of isolates (azithromycin arm and placebo arm). 
following treatment cycles. The effect of any such increase is uncertain. If such transient increases led to increased disease during that time, we would have expected to see an increase in deaths in the azithromycin arm during that small window. However, another study has shown that there were fewer deaths in the period immediately following azithromycin administration. ${ }^{27}$ Further, one would expect that if resistance were to increase, it would be most evident following multiple doses of azithromycin. Although we cannot rule out transient resistance (i.e., observable at times less than 12 months), our findings suggest that the children were recolonized by 12 months to approximate the community prevalence of resistance. As such, treatment of this small subgroup of children compared with the general population may have been insufficient to alter the overall community pattern of resistance. Low rates of resistance following targeted administration of azithromycin was also observed in a study in Nepal, ${ }^{28}$ Our findings could be due to incomplete coverage of children with azithromycin. The mean coverage of communities in the azithromycin arm was 75-78\%; thus, there were some children in the 1- to 59-month age range, most with presumably susceptible strains, which did not contribute to any increase in resistance. Our study, a cluster-randomized trial, was designed to detect an increase in resistance in children living in communities that were randomized to receive either azithromycin or placebo, rather than to evaluate resistance in individual children who had been treated or not treated. From previous research, it is unclear what level of community coverage is needed to drive the increase and durability of resistance, in part because coverage has not been reported or has been reportedly very high in previous studies.

Third, our surveys were cross-sectional representations of resistance in children in the participating communities. Given random sampling of children at the time of survey (with no requirement of previous residency in the community), it is possible that some surveyed children did not receive prior doses of azithromycin, which could have lowered the rates of resistance in the azithromycin arm. Nonetheless, more than half of the sample had received at least three of the four doses at 24 months, suggesting that the composition of the sample was not a major contributing factor.

The study medications were completely masked to the study team and participants, and there is no evidence this was breeched. Therefore, it is unlikely that the results reflect inadvertent receipt of azithromycin in the control communities or vice versa.

A significant limitation to the study is the decline in recovery rates of both organisms (S. pneumoniae and $E$. coli) overtime. In the case of $E$. coli, one would expect isolation rates of $90-100 \%{ }^{29}$; instead, even at baseline, recovery rates were unexpectedly low (62\%). This likely lessened the study's power to detect a difference in prevalence of resistance between the intervention and placebo arms. Nonetheless, the point estimates in the treatment and placebo arms were not markedly dissimilar, offering some plausibility to the findings. In the case of $S$. pneumoniae, the low recovery may be attributed-in part-to pneumococcal vaccination in Kilosa in the year following initiation of our study. Vaccination will affect carriage rates of those $S$. pneumoniae serotypes covered by the conjugate vaccine in use; it will also alter nasopharyngeal colonization both with non-vaccine serotypes and competing flora. ${ }^{30}$ In the absence of serotyping, it is difficult to quantify that effect. Regional studies of S. pneumoniae vaccine coverage before vaccination have shown variable (23-42\%) rates of recovered isolates to be 10-valent pneumococcal conjugate vaccine (PCV10) vaccine serotypes. ${ }^{31,32}$ In Kenya, Hammitt et al. ${ }^{33}$ evaluated annual cross-sectional S. pneumoniae carriage in the 2 years before and after incorporation of the PCV10 into the national immunization program. Among children younger than 5 years, the baseline carriage rates for vaccine and non-vaccine $S$. pneumoniae serotype were $34 \%$ and, $41 \%$ respectively. Subsequent to PCV10 vaccination, the carriage rates were $13 \%$ and $57 \%$, respectively. ${ }^{33}$ Serotyping was not performed in our study and might have offered an insight into the impact of the pneumococcal vaccine on the findings.

Transportation and storage may also have affected recovery rates, despite strict cold chain maintenance, spanning collection, the use of guarded freezers, active monitoring of generators, and transportation of specimens on dry ice to the laboratory at Muhimbili. Nonetheless, we acknowledge the potential impact of transport and storage on labile organisms such as S. pneumoniae. Of note, any external factor that may have impacted the isolation rate had a similar effect in both the intervention and control communities so that an elevated level of resistance-if present-should have been detected even with fewer isolates. Furthermore, we used the most current CLSI guidelines to categorize resistance ${ }^{20}$; differences in break points across guidelines can impact the analysis. Differences in the definition of resistance also account for changes in the reporting; specifically, previous publication of our baseline resistance did not combine intermediate with resistant strains. ${ }^{16}$ Finally, there is a complex interplay between antibiotic resistance and bacterial fitness. ${ }^{34,35}$ Although development of resistance should confer survival advantage in the presence of the cognate drug, this could also come at the cost of a competitive fitness disadvantage during storage, thereby impacting the recovery rates. We note, however, that the isolation rate was not lower in the children in the azithromycin arm, which likely would have been the case if there were more resistant strains that could not be recovered.

In conclusion, a randomized controlled trial in Tanzania did not show an increase in azithromycin resistance within $S$. pneumoniae and $E$. coli isolates after four biannual rounds of treatment. It is important to emphasize that our reporting was confined to only two organisms. Despite their clinical importance, S. pneumoniae and $E$. coli fail to represent the spectrum of organisms that might be susceptible to selection pressure. Although azithromycin resistance in $S$. pneumoniae or $E$. coli does not appear to account for lack of efficacy against child mortality for this site, ${ }^{6}$ the findings should be interpreted together with the acknowledged limitations of this study.

Received January 28, 2019. Accepted for publication August 2, 2019. Published online February 17, 2020.

Acknowledgments: We would like to thank all members of the MORDOR field study team in Kilosa and the technical staff in the microbiology laboratory at the Muhimbili University of Health and Allied Sciences. We also thank the members of the Data and Safety Monitoring Committee, University of Washington, Seattle, WA; Judd L. Walson, Liverpool School of Tropical Medicine, Liverpool, United Kingdom; Allen W. Hightower, Loyola University, Chicago, IL; Emily E. Anderson, Berhan Public Health and Eye Care Consultancy, Addis Ababa, Ethiopia; Wondu Alemayehu, Tulane University, New Orleans, LA; and Latha Rajan. This trial is registered at Clinicaltrials.gov (NCT02047981). 
Financial support: This study was made possible by grants from the Bill \& Melinda Gates Foundation (OPP1032340 and OPP1201895). E. M. B. reports personal fees from Grifols Diagnostics Solutions outside the submitted work. T. M. L. reports grants from BMGF during the conduct of the study.

Disclosure: E. M. B. reports non-financial support from Terumo BCT.

Authors' addresses: Evan M. Bloch, Department of pathology, Johns Hopkins School of Medicine, Baltimore, MD, E-mail: ebloch2@ jhmi.edu. Christian L. Coles, Department of Microbiology, Uniformed Services University of the Health Sciences, Bethesda, MD, E-mail: ccoles@idcrp.org. Mabula Kasubi, Department of Microbiology, Muhimbili University of Health and Allied Sciences, Dar es Salaam, Tanzania, E-mail: mkasubi68@gmail.com. Jerusha Weaver, Beatriz Munoz, and Sheila K. West, Dana Center for Preventive Ophthalmology, Johns Hopkins School of Medicine, Baltimore, MD, E-mails: jerusha.u.weaver@gmail.com, bmunoz@jhmi.edu, and shwest@jhmi.edu. Zakayo Mrango, National Institute for Medical Research, Kilosa, Tanzania, E-mail: mrango@yahoo.com. Thomas M. Lietman, Francis I Proctor Foundation, University of California, San Francisco, San Francisco, CA, E-mail: tom.lietman@ucsf.edu.

This is an open-access article distributed under the terms of the Creative Commons Attribution (CC-BY) License, which permits unrestricted use, distribution, and reproduction in any medium, provided the original author and source are credited.

\section{REFERENCES}

1. Coles CL, Levens J, Seidman JC, Mkocha H, Munoz B, West S, 2012. Mass distribution of azithromycin for trachoma control is associated with short-term reduction in risk of acute lower respiratory infection in young children. Pediatr Infect Dis $\mathrm{J}$ 31: 341-346.

2. Montero L, 1996. A comparative study of the efficacy, safety and tolerability of azithromycin and cefaclor in the treatment of children with acute skin and/or soft tissue infections. $J$ Antimicrob Chemother 37 (Suppl C): 125-131.

3. Coles CL, Seidman JC, Levens J, Mkocha H, Munoz B, West S, 2011. Association of mass treatment with azithromycin in trachoma-endemic communities with short-term reduced risk of diarrhea in young children. Am J Trop Med Hyg 85: 691-696.

4. Adachi JA, Ericsson CD, Jiang ZD, DuPont MW, MartinezSandoval F, Knirsch C, DuPont HL, 2003. Azithromycin found to be comparable to levofloxacin for the treatment of US travelers with acute diarrhea acquired in Mexico. Clin Infect Dis 37: 1165-1171.

5. Melese $\mathrm{M}$ et al., 2004. Feasibility of eliminating ocular Chlamydia trachomatis with repeat mass antibiotic treatments. JAMA 292: 721-725.

6. Keenan JD et al., 2018. Azithromycin to reduce childhood mortality in sub-Saharan Africa. N Engl J Med 378: 1583-1592.

7. Doern GV, Heilmann KP, Huynh HK, Rhomberg PR, Coffman SL, Brueggemann AB, 2001. Antimicrobial resistance among clinical isolates of Streptococcus pneumoniae in the United States during 1999-2000, including a comparison of resistance rates since 1994-1995. Antimicrob Agents Chemother 45: 1721-1729.

8. Doern GV, Richter SS, Miller A, Miller N, Rice C, Heilmann K, Beekmann S, 2005. Antimicrobial resistance among Streptococcus pneumoniae in the United States: have we begun to turn the corner on resistance to certain antimicrobial classes? Clin Infect Dis 41: 139-148.

9. Batt SL, Charalambous BM, Solomon AW, Knirsch C, Massae PA, Safari S, Sam NE, Everett D, Mabey DC, Gillespie SH, 2003. Impact of azithromycin administration for trachoma control on the carriage of antibiotic-resistant Streptococcus pneumoniae. Antimicrob Agents Chemother 47: 2765-2769.

10. Haug $S$ et al., 2010. The decline of pneumococcal resistance after cessation of mass antibiotic distributions for trachoma. Clin Infect Dis 51: 571-574.

11. Gaynor BD, Holbrook KA, Whitcher JP, Holm SO, Jha HC, Chaudhary JS, Bhatta RC, Lietman T, 2003. Community treatment with azithromycin for trachoma is not associated with antibiotic resistance in Streptococcus pneumoniae at 1 year. $\mathrm{Br} \mathrm{J} \mathrm{Oph}$ thalmol 87: 147-148.
12. Skalet AH et al., 2010. Antibiotic selection pressure and macrolide resistance in nasopharyngeal Streptococcus pneumoniae: a cluster-randomized clinical trial. PLoS Med 7: e1000377.

13. Coles CL, Mabula K, Seidman JC, Levens J, Mkocha H, Munoz B, Mfinanga SG, West S, 2013. Mass distribution of azithromycin for trachoma control is associated with increased risk of azithromycin-resistant Streptococcus pneumoniae carriage in young children 6 months after treatment. Clin Infect Dis 56: 1519-1526.

14. O'Brien KS, Emerson P, Hooper PJ, Reingold AL, Dennis EG, Keenan JD, Lietman TM, Oldenburg CE, 2019. Antimicrobial resistance following mass azithromycin distribution for trachoma: a systematic review. Lancet Infect Dis 19: e14-e25.

15. Leach AJ, Shelby-James TM, Mayo M, Gratten M, Laming AC, Currie BJ, Mathews JD, 1997. A prospective study of the impact of community-based azithromycin treatment of trachoma on carriage and resistance of Streptococcus pneumoniae. Clin Infect Dis 24: 356-362.

16. Bloch EM, West SK, Mabula K, Weaver J, Mrango Z, Munoz B, Lietman T, Coles C, 2017. Antibiotic resistance in young children in Kilosa district, Tanzania 4 Years after mass distribution of azithromycin for trachoma control. Am J Trop Med Hyg 97: 815-818.

17. Littmann J, Buyx A, Cars O, 2015. Antibiotic resistance: an ethical challenge. Int J Antimicrob Agents 46: 359-361.

18. Mendelson M, Rottingen JA, Gopinathan $U$, Hamer DH, Wertheim $\mathrm{H}$, Basnyat B, Butler C, Tomson G, Balasegaram M, 2016. Maximising access to achieve appropriate human antimicrobial use in low-income and middle-income countries. Lancet 387: 188-198.

19. Littmann J, Viens AM, 2015. The ethical significance of antimicrobial resistance. Public Health Ethics 8: 209-224.

20. CLSI, 2018. Performance Standards for Antimicrobial Susceptibility Testing; Twenty-Eighth Informational Supplement. CLSI Document M100-S28. Wayne, PA: CLSI.

21. Seidman JC, Coles CL, Silbergeld EK, Levens J, Mkocha H, Johnson LB, Munoz B, West SK, 2014. Increased carriage of macrolide-resistant fecal $E$. coli following mass distribution of azithromycin for trachoma control. Int $J$ Epidemiol 43: 1105-1113.

22. Maher MC, Alemayehu W, Lakew T, Gaynor BD, Haug S, Cevallos V, Keenan JD, Lietman TM, Porco TC, 2012. The fitness cost of antibiotic resistance in Streptococcus pneumoniae: insight from the field. PLoS One 7: e29407.

23. Bartoloni A et al., 2008. Increasing resistance in commensal Escherichia coli, Bolivia and Peru. Emerg Infect Dis 14: 338340.

24. Kalter HD, Gilman RH, Moulton LH, Cullotta AR, Cabrera L, Velapatino B, 2010. Risk factors for antibiotic-resistant Escherichia coli carriage in young children in Peru: community-based cross-sectional prevalence study. Am J Trop Med Hyg 82: 879-888.

25. Wright GD, 2007. The antibiotic resistome: the nexus of chemical and genetic diversity. Nat Rev Microbiol 5: 175-186.

26. Phuc Nguyen $M C$, Woerther $P L$, Bouvet $M$, Andremont $A$, Leclercq R, Canu A, 2009. Escherichia coli as reservoir for macrolide resistance genes. Emerg Infect Dis 15: 1648-1650.

27. Porco TC et al., 2019. Mass oral azithromycin for childhood mortality: timing of death after distribution in the MORDOR trial. Clin Infect Dis 68: 2114-2116.

28. Fry AM, Jha HC, Lietman TM, Chaudhary JS, Bhatta RC, Elliott J, Hyde T, Schuchat A, Gaynor B, Dowell SF, 2002. Adverse and beneficial secondary effects of mass treatment with azithromycin to eliminate blindness due to trachoma in Nepal. Clin Infect Dis 35: 395-402.

29. Gottesman T, Yossepowich O, Harari-Schwartz O, Tsivian A, Idler J, Dan M, 2015. The value of rectal cultures in treatment of sepsis following post-transrectal ultrasound-guided prostate biopsy. Urol Int 95: 177-182.

30. Devine VT, Jefferies JM, Clarke SC, Faust SN, 2015. Nasopharyngeal bacterial carriage in the conjugate vaccine era with a focus on pneumococci. J Immunol Res 2015: 394368.

31. Lindstrand A, Kalyango J, Alfven T, Darenberg J, Kadobera D, Bwanga F, Peterson S, Henriques-Normark B, Kallander K, 2016. Pneumococcal carriage in children under five years in 
Uganda-will present pneumococcal conjugate vaccines be appropriate? PLoS One 11: e0166018.

32. Nackers $F$ et al., 2017. Carriage prevalence and serotype distribution of Streptococcus pneumoniae prior to 10-valent pneumococcal vaccine introduction: a population-based cross-sectional study in South Western Uganda, 2014. Vaccine 35: 5271-5277.

33. Hammitt LL et al., 2014. Population effect of 10-valent pneumococcal conjugate vaccine on nasopharyngeal carriage of
Streptococcus pneumoniae and non-typeable Haemophilus influenzae in Kilifi, Kenya: findings from cross-sectional carriage studies. Lancet Glob Health 2: e397-e405.

34. Roux D et al., 2015. Fitness cost of antibiotic susceptibility during bacterial infection. Sci Transl Med 7: 297ra114.

35. Geisinger E, Isberg RR, 2017. Interplay between antibiotic resistance and virulence during disease promoted by multidrugresistant bacteria. J Infect Dis 215 (Suppl 1): S9-S17. 